\title{
The Correct Replacement for the Wrong Starling's law is the Hydrodynamic of the Porous Orifice (G) Tube: The Complete Physics and physiological Evidence with Clinical Relevance and Significance
}

\author{
Ahmed N Ghanem
}

Mansoura University, Retired Consultant Urological Surgeon, No 1, *Corresponding author

Ahmed N. Ghanem, Mansoura University, Retired Consultant Urological Surgeon, No 1, Jasmine Tower, President Mubarak Street, Mansoura 35511, Egypt Jasmine Tower, President Mubarak Street, Mansoura 35511, Egypt

Submitted: 13 Jan 2020; Accepted: 20 Jan 2020; Published: 17 Feb 2020

\begin{abstract}
Introduction and objective: To report the complete evidence that Starling's law is wrong and the correct replacement is the hydrodynamic of the G tube. New physiological evidence is provided with clinical relevance and significance.

Material and methods: The physics proof is based on G tube hydrodynamic. Physiological proof is based on study of the hind limb of sheep: running plasma and later saline through the artery compared to that through the vein as regards the formation of oedema. The clinical significance is based on 2 studies one prospective and a 23 case series on volumetric over load shocks (VOS).
\end{abstract}

Results: Hydrodynamic of G tube showed that proximal, akin to arterial, pressure induces suction "absorption" not "filtration". In Poiseuille's tube side pressure is all positive causing filtration based on which Starling proposed his hypothesis, The physiological evidence proves that the capillary works as G tube not Poiseuille's tube: Oedema occurred when fuids are run through the vein but not through the artery. There was no difference using saline or plasma proteins. The wrong Starling's law dictates the faulty rules on fluid therapy inducing VOS and causing ARDS.

Conclusion: Hydrodynamic of the G tube challenges the role attributed to arterial pressure as filtration force in Starling's law. A literature review shows that oncotic pressure does not work either. The new hydrodynamic of G tube is proposed to replace Starling's law which is wrong on both forces. The physiological proof and relevance to clinical importance on the pathogenesis of clinical syndromes are discussed.

Keywords: Capillary physiology; hydrodynamics; Starling's law; fluid therapy; shock; hyponatramia; the acute respiratory distress syndrome (ARDS); The multiple vital organ dysfunction/ failure syndrome; (MVOD/F); Acute kidney injury (AKI)

\section{Abbreviations}

VO: Volumetric overload

VOS: Volumetric overload shocks

VOS1: Volumetric overload shock, Type 1

VOS2: Volumetric overload shock, Type2

TURS: The transurethral resection of the prostate syndrome

ARDS: The adult respiratory distress syndrome

MVOD/F: The multiple vital organ dysfunction/ failure syndrome

AKI: Acute kidney injury

HN: Hyponatraemia

BP: Arterial Blood pressure

CVP: Central venous pressure
ISF: Interstitial fluid

G Tube: The Porous Orifice Tube

PP: Proximal pressure to the $\mathrm{G}$ tube akin to arterial Blood pressure

DP: Distal Pressure to the G tube akin to venous pressure

LP: Lumen pressure of the $G$ tube

FP: Flow pressure is the positive pressure inside the G Tube

SP: Side pressure is the negative pressure on the wall of the G Tube

\section{Introduction}

Literature review on capillary physiology and ultra-structure that has been previously reported demonstrated that albumen's oncotic pressure as re-absorption force does not work in vivo; neither in clinical practice nor in physiology. This is one of the two forces of Starling's law has proved wrong. My research work proves that the other Starling's law force of hydrostatic pressure causing filtration is also wrong. The clinical relevance and significance in relation to the pathogenesis and therapy of the transurethral resection of the 
prostate (TURP) syndrome, acute dilution hyponatraemia (HN), the acute respiratory distress syndrome (ARDS) or the multiple vital organ dysfunction/ failure (MVOD/F) syndrome and acute kidney injury (AKI) in relation to its patho-aetiology of volumetric overload shocks (VOS) are discussed. The key points of the complete enlightening evidence are summarised here:-

1. Starling's hypothesis became a law prior to the discovery of the capillary ultra-structure and the correct physiology $[1,2]$.

2. The capillary has a pre-capillary sphincter as reported by Rhodin in 1967 which makes it different from Poiseuille's tube of uniform diameter as my research demonstrated [3].

3. The capillary has porous wall of intercellular slits that allow the passage of plasma proteins as shown by Karnoveski in 1967 [4]. Hence plasma proteins cannot exert an oncotic pressure in vivo.

4. The osmotic chemical composition of various body fluids is identical to plasma proteins as demonstrated by Hendry in 1962 [5]. Plasma protein, if it works, is too weak and too slow to effect fluid absorption.

5. Guyton and Coleman (1968) demonstrated that the interstitial fluid (ISF) space has a negative pressure of $-7 \mathrm{~cm}$ water and Calnan et al (1972) showed that the lymph has the same negative pressure $[6,7]$. The pressure under the skin is negative.

6. The oncotic pressure of plasma proteins does not work as absorption force neither in physiology as proved by Hendry in 1962 nor in clinical practice demonstrated by Cochrane Injuries Group in 1998 [5,8].

7. Inadequacy in explaining the capillary-ISF transfer in many parts of the body as reported by Keele et al in 1982, particularly vital organs, has previously called for reconsideration of Starling's hypothesis by Renkin in $1984[9,10]$.

8. My research work has demonstrated that the hydrostatic or rather the dynamic "arterial" pressure does not cause filtration across the wall of porous orifice $(\mathrm{G})$ tube as proposed by Starling. It causes suction.

9. This pressure induces negative side pressure gradient along the $\mathrm{G}$ tube causing suction maximum near the inlet and turns positive causing filtration maximum near the exit as based on physics experiments on the $\mathrm{G}$ tube and physiological research on the hind limb of sheep [11-13].

10. The physiological study on the hind limb of sheep have completed the evidence that Starling's law is wrong as the capillary works as G tube not Poiseulli's tube [13].

11. Starling's law being wrong underlies all errors and misconceptions on fluid therapy misleading physicians into giving too much fluid during resuscitation of shock and the acutely ill patients and during prolonged surgery thus inducing VOS and causing ARDS or MVOD/F and AKI [14].

Here all the complete evidence based on physics and physiological investigations that Starling's law is wrong on both forces and the correct replacement is the hydrodynamics of the $\mathrm{G}$ tube is summarised.' Such evidence was reported as early as 1988 as appendix in MD Thesis and 1990 in the discussion of an article $[15,16]$. This physics evidence was preliminary reported in 2001 and concluded in 2017 [11,12]. The physiological evidence was reported in in 2017 [13]. The complete evidence with the clinical relevance and significance of discovering volumetric overload shocks (VOS) inducing ARDS or MVOD/F and AKI is finalised here and now in 2020 [17-21].

\section{Materials and Methods}

The complete physics and physiological evidence and the clinical relevance and significance are based on:

1. Analytical literature review of articles on the capillary ultrastructure and physiology.

2. Physics study on the hydrodynamic of $\mathrm{G}$ tube. Using porous tubes with narrow inlet and measuring the dynamic components of lumen pressure (LP), which are the flow pressure (FP) in lumen and side pressures (SP) exerted on its wall. The factors that affect $\mathrm{SP}$ including proximal pressure (PP) akin to arterial pressure, distal pressure (DP) akin to venous pressure and the size of the orifice of the $\mathrm{G}$ tube were evaluated.

3. Physiological study on the hind limb of sheep running saline and later albumen through the artery and comparing it when fluid is run through the vein as regards to the formation of oedema or accumulation of fluid under the skin or cling membrane.

4. Prospective clinical study on 100 consecutive transurethral resection of the prostate (TURP) patients among whom 10 developed the TURP syndrome randomized into treatments comparing hypertonic sodium therapy (HST) with the conservative treatment of using volume expansion for treating the new shock of VOS.

5. Case series of 23 patients who suffered the TURP syndrome. The first 3 patients were wrongly diagnosed being mistaken for a known shock and treated with volume expansion causing ARDS and death. The remaining 20 patients were correctly diagnosed as VOS and treated with $\mathrm{HST}$ of $5 \% \mathrm{NaCl}$ or $8.4 \% \mathrm{NaCo} 3$ were saved from certain death.

6. Critical comprehensive literature review of the literature on the TURP syndrome, HN, ARDS and fluid therapy in hospitals.

\section{Results}

The results of physics experiments demonstrate that the hydrostatic or rather the dynamic pressure in a porous orifice $(\mathrm{G})$ tube induced by the high positive proximal pressure, akin to arterial pressure, induces a negative side pressure gradient on the tube's wall. This SP is negative maximum at orifice (Figure 1) and turns positive at the distal part maximum near the exit (Figure 2). This produces the phenomenon of the $G$ tube; fluid flows out from side holes near the outlet or the 'venous' end while it flows in by suction through holes near the inlet or 'arterial' end, re-entering back into the lumen of the $\mathrm{G}$ tube. Fluid around the $\mathrm{G}$ tube in chamber $\mathrm{C}$ moves in an opposite direction to flow inside its lumen in a magnetic field-like pattern (Figure 3).

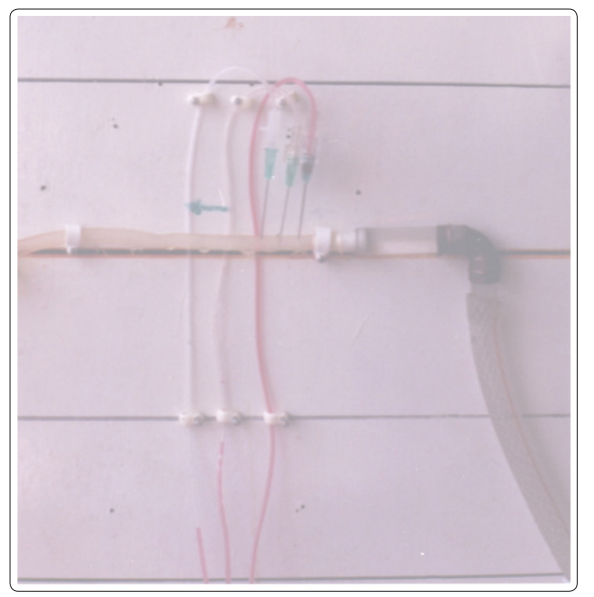

Figure 1: Perpendicular needles inserted into a rubber orifice tube at 10, 20 and $30 \mathrm{~mm}$ distance from the orifice, with bevels facing 
downstream, demonstrate the negative energy SP gradient along the proximal part of the tube by the sucked columns of fluids in manometer tubes from a jar $300 \mathrm{~mm}$ below the tube. (Reproduced with permission of the author from $\mathrm{BHC}$ open access journal; reference 12)

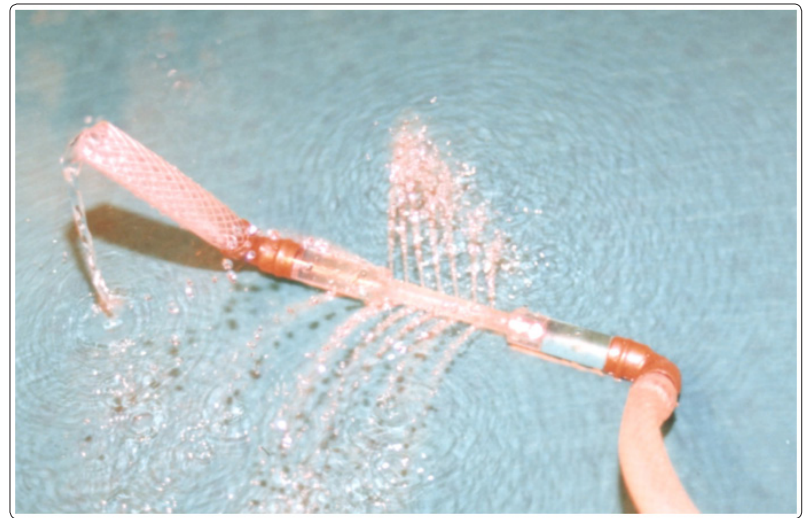

Figure 2: Fluid flows out autonomously through distally situated side holes of a porous orifice $(\mathrm{G})$ tube where SP is positive. Air suction occurs through side holes of the proximal part, as shown in Figure 1 , but is not seen here. The fluid around the $\mathrm{G}$ tube has magnetic field shape shown above the $\mathrm{G}$ tube. (Reproduced with permission of the author from BHC open access journal; reference 12)

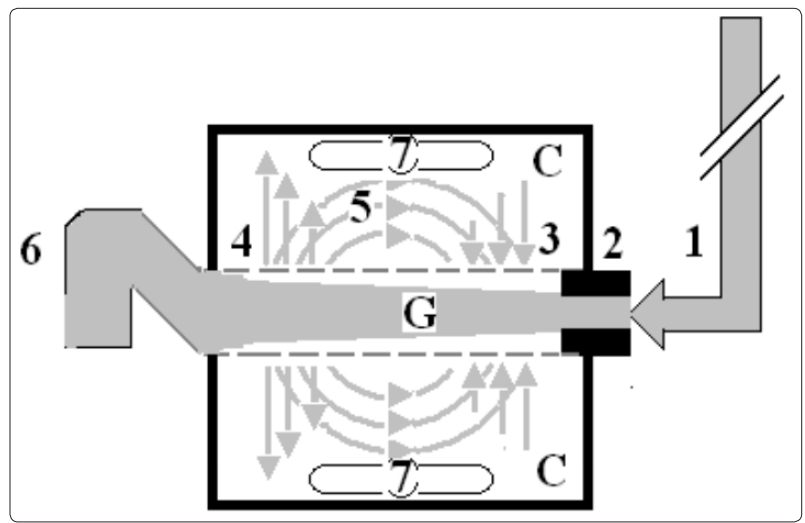

Figure 3: Shows diagram of the porous orifice $(G)$ tube enclosed in chamber $(\mathrm{C})$ based on several photographs, some of it are shown here, demonstrating the magnetic field-like G-C circulation phenomenon. The proximal inflow (arterial) pressure (1) pushes fluid through the orifice (2) creating fluid jet in the lumen of the G tube. The fluid jet creates negative side pressure gradient causing suction maximal over the proximal half of the G tube near the inlet (3) that sucks fluid into lumen. The side pressure gradient turns positive pushing fluid out of lumen over the distal half maximally near the outlet (4). Thus the fluid around $\mathrm{G}$ tube inside $\mathrm{C}$ moves in magnetic field-like fluid circulation (5) taking an opposite direction to lumen flow of $\mathrm{G}$ tube. The inflow (arterial) pressure (1) and orifice (2) induce the negative side pressure energy creating the dynamic G-C circulation phenomenon that is rapid, autonomous and efficient in moving fluid out from the $\mathrm{G}$ tube lumen at (4), irrigating $\mathrm{C}$ at (5), then sucking it back again at (3), maintaining net negative energy pressure (7) inside C. The distal outflow (venous) pressure (6) enhances outflow at (4) and its elevation may turn the negative energy pressure (7) inside $\mathrm{C}$ into positive, increasing volume and pressure inside $\mathrm{C}$ chamber. (Reproduced with permission of the author from BHC open access journal; reference 12)
This $\mathrm{G}-\mathrm{C}$ circulation phenomenon also induces a net negative pressure in chamber (CP), surrounding the $\mathrm{G}$ tube, demonstrated in (Figures 4 and 5). A negative pressure also occurs inside chamber $\mathrm{C}$, akin to that of the ISF space, causing fluid to move in an opposite direction to the main flow in the $\mathrm{G}$ tube lumen.

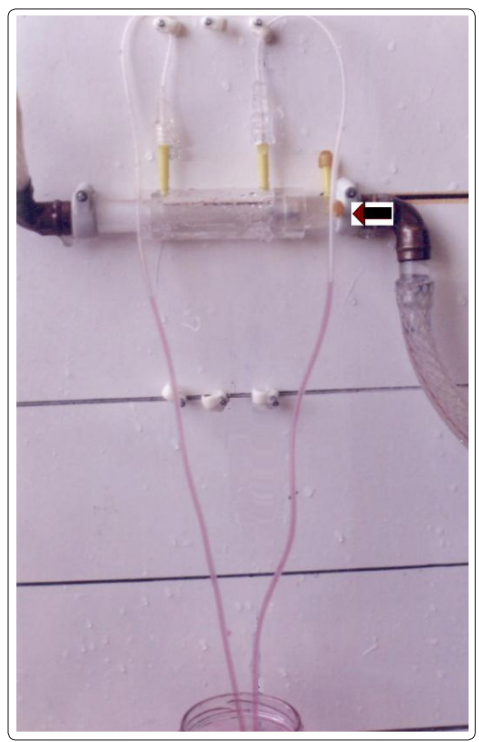

Figure 4: The net negative pressure of a closed chamber (CP) surrounding the $\mathrm{G}$ tube, is demonstrated by the sucked fluid in two vertical manometer tubes from a jar $300 \mathrm{~mm}$ below. (Reproduced with permission of the author from $\mathrm{BHC}$ open access journal; reference 12)

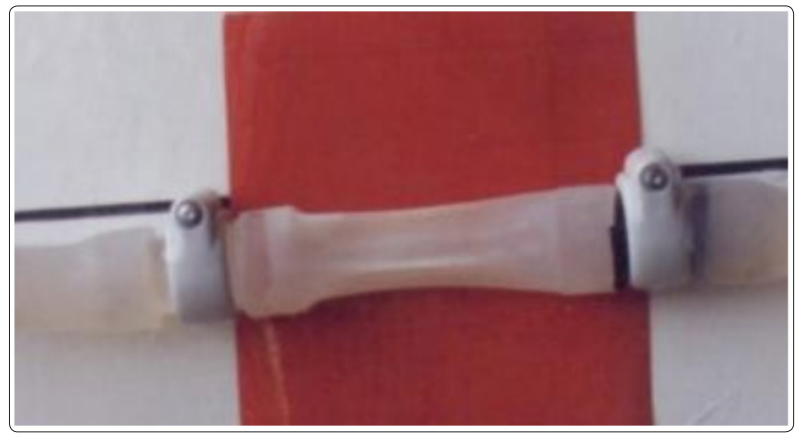

Figure 5: The negative CP (Figure 4) collapses a membrane around the $\mathrm{G}$ tube. This means that the $\mathrm{G}$ tube irrigates $\mathrm{C}$ chamber well under negative pressure without accumulation of fluid. In the case of capillaries shown on the physiological investigation on the hind limb of sheep (see text) arterial flow irrigates ISF space and cells well under negative pressure without odema formation. While venous flow induces oedema irrespective whether saline or plasma proteins were used. (Reproduced with permission of the author from BHC open access journal; reference 12)

The physiological study on the hind limb of sheep running saline and comparing it to albumen first through the artery and later through vein as regards the formation of oedema or accumulation of fluid under cling membrane that replaced the skin. Oedema occurred only when fluid is run through the vein and not through the artery. This means that the capillary works as the G tube not Poiseuille's tube. The G tube phenomenon irrigates ISF space and cells well without oedema formation. There was no difference on running plasma from running saline as circulatory fluid; both fluids induced oedema when 
run through the vein but not when run through the artery.

The prospective study demonstrated that volumetric overload (VO) is the most significant factor in inducing the vascular shock of TURP syndrome (Figure 6 and Table 1). This shock is recognized as VOS. The correct lifesaving treatment for VOS1 of the TURP syndrome is hypertonic sodium therapy (HST) of $5 \% \mathrm{NaCl}$ or $8.4 \% \mathrm{NaCo} 3$.

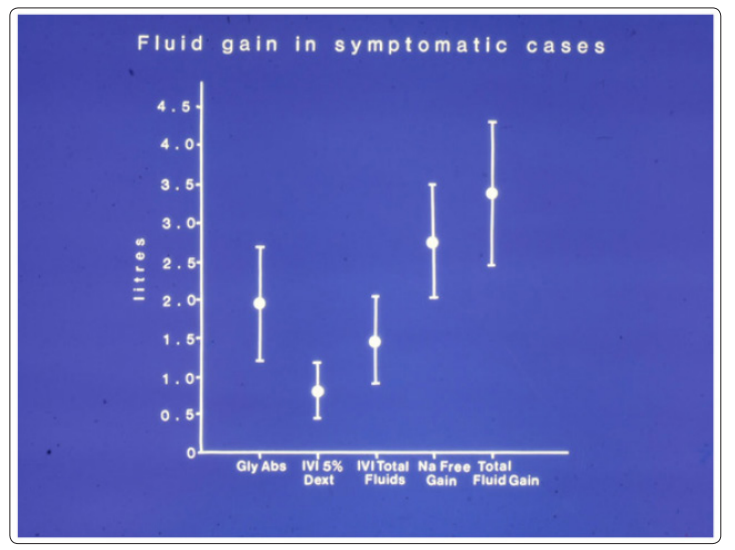

Figure 6: Shows the means and standard deviations of volumetric overload in 10 symptomatic patients presenting with shock and hyponatraemia among 100 consecutive patients during a prospective study on transurethral resection of the prostate. The fluids were of Glycine absorbed (Gly abs), intravenously infused 5\% Dextrose (IVI Dext) Total IVI fluids, Total Sodium-free fluid gained (Na Free Gain) and total fluid gain in litres. (Reproduced with permission of the author and Editor of BJU Int.; reference 16)

The case series of 23 patients demonstrated that mistaking VOS for one of the recognized shocks such as septic or haemorrhagic shock and treating it with further volume expansion caused ARDS and death of the first 3 patients. Diagnosing VOS correctly and treating it with prompt HST saved the lives of 20 patients. The volumetric overload in these 23 cases is shown in (Figure 7).

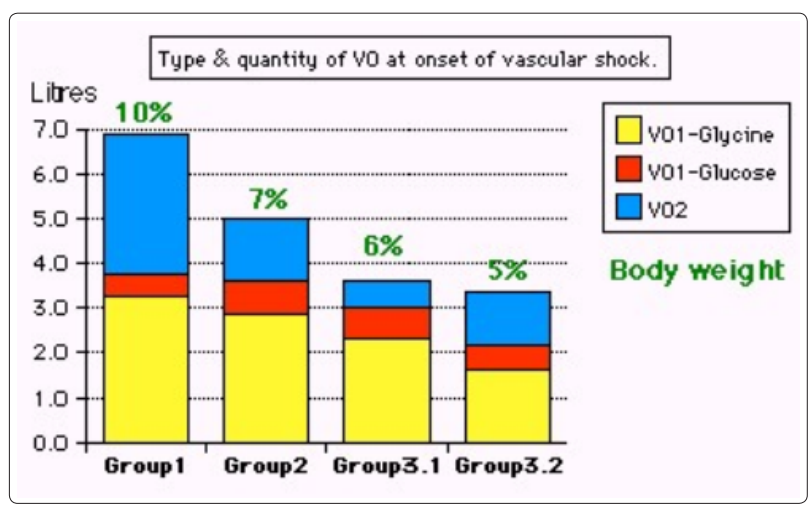

Figure 7: Shows volumetric overload (VO) quantity (in litres and as percent of body weight) and types of fluids. Group 1 was the 3 patients who died in the case series as they were misdiagnosed as one of the previously known shocks and treated with further volume expansion. Group 2 were 10 patients from the series who were correctly diagnosed as volumetric overload shock and treated with hypertonic sodium therapy (HST). Group 3 were 10 patients who were seen in the prospective study and subdivided into 2 groups; Group 3.1 of 5 patients treated with HST and Group 3.2 of 5 patients who were treated with guarded volume expansion using isotonic saline. (Reproduced with permission of the author from BHC open access journal; reference 18)

VOS is of two types depending on the type of fluid inducing it. Sodium-free fluid such as $1.5 \%$ Glycine and 5\% Glucose induce VOS1. The TURP syndrome and dilution hyponatraemia (HN) represent VOS1. Sodium-based fluid such as Normal Saline, Hartmann' solution, plasma, plasma substitute and/or blood induce VOS2. Both VOS are always mistaken for one of the recognized shocks and wrongly treated with volume expansion using isotonic sodium-based fluids causing ARDS and death. Discovery of VOS has resolved the puzzles of TURP syndrome, HN and ARDS or $\mathrm{MVOD} / \mathrm{F}$ syndrome, discovering its exact patho-aetiology and successful lifesaving therapy.

\section{Discussion}

The presented results demonstrate that the hydrodynamics of the $\mathrm{G}$ tube are different from that of Hagen (1839)-Poiseuille's tube (1840), which in turn challenge the role attributed to arterial pressure as the filtration force in the capillary circulation as proposed by Starling [1]. Inflow PP induces a fluid jet with LP inside both the G tube and Poiseuille's tubes, which has the dynamic FP and SP components. Over the proximal part of the $\mathrm{G}$ tube, the net effect of the negative SP gradient is suction (Figure 1) and turns positive causing filtration near the exit but in Poiseuille's tube it is filtration all along the entire length of the tube. Both the dynamic FP and SP components of a fluid jet are extensions of the well known Bernoulli (1738) and Venturi effects.

The major difference between Poiseuille's tube and G tube, is that the first induces an all positive pressure gradient which can only cause filtration along the entire length of a tube - hence it requires another force for re-absorption. The $\mathrm{G}$ tube has both its negative and positive dynamic pressure components which autonomously induce both absorption (Figure 1) and filtration (Figure 2) through the $\mathrm{G}-\mathrm{C}$ circulation phenomenon (Figure 3 ) and the net negative $\mathrm{CP}$ in a surrounding fluid chamber $\mathrm{C}$ (Figures 4 and 5). Physics of the $\mathrm{G}-\mathrm{C}$ phenomenon [11,12], with physiological evidence [13] and clinical relevance and significance inducing volumetric overload shocks (VOS) [14-19] and causing ARDS or MVOD/F syndrome and AKI [20,21] are discussed Vide Infra.

Dr Starling proposed his hypothesis $>80$ years prior to the discovery of the capillary ultrastructure and correct physiology which are as follows. He based his hypothesis on Poiseuille's work in which the hydrostatic pressure is a positive function of the arterial pressure causing filtration, but in the $\mathrm{G}$ tube hydrodynamic as a porous orifice tube this pressure is different causing suction. Thus Starling's low is wrong on both forces because

1. The capillary has a pre-capillary sphincter as reported by Rhodin in 1967 which makes it different from Poiseuille's tube of uniform diameter as my research demonstrated [3].

2. The capillary has porous wall of intercellular slits that allow the passage of plasma proteins as shown by Karnoveski in 1967 [4]. Hence plasma proteins cannot exert an oncotic pressure in vivo.

3. The osmotic chemical composition of various body fluids is identical to plasma proteins as demonstrated by Hendry in 1962, Hence oncotic pressure if it exists is too week and too slow force to cause absorption [5].

4. The oncotic pressure of plasma proteins does not work as 
absorption force neither in physiology as proved by Hendry in 1962 nor in clinical practice demonstrated by Cochrane Injuries Group in 1998 [5,10].

5. Guyton and Coleman (1968) demonstrated that the interstitial fluid (ISF) space has a negative pressure of $-7 \mathrm{~cm}$ water and Calnan et al (1972) showed that the lymph has the same negative pressure $[6,7]$. The pressure under the skin is negative. That cannot be explained by Starling's law.

6. Inadequacy in explaining the capillary-ISF transfer in many parts of the body as reported by Keele et al in 1982, particularly vital organs, has previously called for reconsideration of Starling's hypothesis by Renkin in 1984 [8,9].

7. My research work reported here demonstrates that the hydrostatic or rather dynamic "arterial" pressure does not cause filtration across the wall of porous orifice $(G)$ tube as proposed by Starling. It causes suction.

8. This pressure induces negative side pressure gradient along the $G$ tube causing suction maximum near the inlet and turns positive maximum near the exit causing filtration as based on physics experiments and physiological research [11-13]. Venous pressure enhances filtration and causes oedema but arterial pressure does not- it causes absorption by suction.

9. The physiological study on the hind limb of sheep has completed the evidence that Starling's law is wrong as the capillary works as G tube not Poiseulli's tube [13].

10. Starling's law being wrong underlies all errors and misconceptions on fluid therapy misleading physicians into giving too much fluid during resuscitation of shock and the acutely ill patients and during prolonged surgery which induce VOS causing ARDS [14-19].

11. Received thinking that elevating central venous pre3ssure (CVP) is synonymous with elevating arterial pressure is prevailing in current clinical practice during fluid therapy for shock and the management of the acutely ill patients. This may be correct during restoration therapy for hypovolemic and haemorrhagic shock, but vascular expansion or volumetric overload (VO) is a different issue as it induces VOS and ARDS or MVOD/F syndrome and AKI [14-21].

12. Persistent attempts to elevate CVP up to levels of 18 to $22 \mathrm{~cm}$ water are common received practice, but wrong because the normal CVP is 0 and most textbooks report a range of -7 to $+7 \mathrm{~cm}$ water $[9,22]$.

13. Clinical observations demonstrate that, in addition to the well known effect of high venous pressure causing oedema, arterial hypertension has no such effect, if not the exact opposite. In clinical practice, although arterial hypertension is common, ISF oedema is unknown among its complications.

14. In the $\mathrm{G}-\mathrm{C}$ model, a minor increase in DP increases fluid volume in chamber $\mathrm{C}$ reverting $\mathrm{CP}$ from negative to positive while slowing the $\mathrm{G}-\mathrm{C}$ circulation $[11,12]$. Increasing DP has similar effect to decreasing $\mathrm{PP}$ on the $\mathrm{G}-\mathrm{C}$ circulation and chamber pressure and volume.

15. Vascular expansion causes VO shocks [14-19]. There is no doubt that the erroneous Starling's law is responsible for the many errors and misconceptions prevailing on fluid therapy for shock and the acutely ill patients which mislead physicians into giving too much fluid that induce VOS causing MVOD/F syndrome or (ARDS and AKI [14-21].

The physiological proof that the capillary works as $\mathrm{G}$ tube A physiological study was conducted on the hind limb of sheep brought from the Butcher shop and wrapped in a cling film to replace the skin. Both normal saline and plasma proteins were used as circulatory fluid, and compared [13]. An electric pump was used to circulate fluid once through the main artery and compared it to another running fluid through the main vein. The formation of oedema was observed by the increase in the limp weight and accumulation of fluid under the cling film that replaced the skin. Here is the abstract of this physiological study.

\section{Objective}

To report physiological evidence that the capillary works as a porous orifice $(\mathrm{G})$ tube proving that Starling's law is wrong.

\section{Material and Methods}

The $G$ tube has a negative side pressure (SP) exerted on its wall. The $G$ tube is akin to capillary and when enclosed in a chamber $(C)$, akin to interstitial fluid space, demonstrating the G-C circulation phenomenon (Figure 3).

Here we report the results of experiments on the hind limb of sheep; first when the fluid is passed through the artery and later when passed through the vein. We monitored the occurrence of oedema of the limb comparing the two circulations. We used normal saline and compared it to plasma proteins in different set of experiments.

\section{Results}

The inflow pressure induces negative SP in the G tube which is responsible for absorption and G-C phenomenon: an autonomous magnetic field-like $\mathrm{G}-\mathrm{C}$ circulation occurs between fluid in the $\mathrm{G}$ tube's lumen and a surrounding fluid compartment $\mathrm{C}$. The hind limb acted as normal without oedema and has a negative interstitial (ISF) pressure when the fluid is passed through the artery i.e. acting like the $G$ tube. There was gross oedema with increase in weight of the limb when the fluid was passed through the vein when the capillary acts like Poiseuille tube. There was no difference between using saline or plasma proteins.

\section{Conclusion}

The reported studies affirm that the capillary works as G tube effecting circulation with ISF which causes negative ISF pressure. When the circulation is reversed through the vein it acted like Poiseuille's tube causing gross oedema and positive ISF pressure. This is the physiological proof that Starling's law is wrong."

The clinical relevance and significance of correcting Starling's law Over 32 years of my career life I did many studies, some reported as communications, MD Thesis and articles [15-21, 23-29]. A summary of some overlooked issues, new concepts and discoveries made since my initial brain storming thoughts were reported at The BMJ and The Lancet is mentioned here [23-25]. The shock that complicates overzealous fluid therapy during poly-trauma resuscitation, recognized as VOS2, may complicate various recognized shocks, diverse diseases requiring fluid therapy or parentral nutrition on intensive care units and during prolonged major surgery [30-42]. The prevalence of morbidity and mortality of this condition known as ARDS is staggering yet if it has attracted a fraction of the attention given to AIDS, it should have been resolved by now [40].

There is no doubt that correcting the faulty Starling's law has led to the discovery of volumetric overload shocks (VOS) that complicate iatrogenic overzealous fluid infusion inducing diverse clinical 
presentations depending on the type of fluid gained, volume and time [14-19]. The severity of the signs is directly proportional to the $\mathrm{VO}$ but inversely to time (VO/T). Fluid type determines the changes of serum solutes and presentation; sodium-free fluids (VO1) inducing VOS1 are characterized with hyponatraemia $(\mathrm{HN})$ with a primary nadir proportional to severity occurring during or immediately after surgery as dilution of the extracellular fluid $[15,16]$.

The gain of VO1 such as 5\% Dextruse, and 1.5\% Glycine may occur either via excessive infusion or inadvertent absorption of irrigating fluids used in endoscopic surgery- known as TURP syndrome or hyponatraemic shock $[15,16,31,35,36,38,39]$. This shock was even first reported as experimentally induced in in dogs [32]. It also affects women undergoing trans-cervical endometrial resection and other endoscopic surgery [37]. Other patients including women and children infused with excessive VO1 fluids such as 5\% dextrose were reported [38,39]. These are examples of VOS type 1 (VOS1). Correcting Starling's law has not only identified the exact pathoaetiology of the TURP syndrome and acute hyponatraemia (HN) but also has rejuvenated a highly successful lifesaving therapy for it; namely; hypertonic sodium therapy (HST) of $5 \% \mathrm{NaCl}$ or $8.4 \% \mathrm{NaCo} 3$.

This VOS1 is characterized by the marker of HN presents with cardiovascular shock. It may present with cardiac arrest or respiratory arrest in isolation or a combination of both "cardiopulmonary arrest" to anaesthetists and surgeons in theatre and with coma to physicians later [14-19,30-33,37-39]. Manifestations of respiratory, cardiac, renal and hepatic dysfunction or failures are also evident and are recognized as MVOD/F syndrome which is the new name for ARDS [20,21,41]. Acute kidney injury (AKI) prevents urinary excretion and sodium loss, so serum HN in VO1 shock is mainly dilution. The hypotension of VO1 is usually mistaken for known shocks of haemorrhage or septicaemia [17-19]. Hence, it is wrongly treated with further vascular expansion using crystalloids, colloids or substitutes and blood. Such VO1 shock and its hypertonic sodium therapy (HST) of $5 \% \mathrm{NaCl}$ was reported 7 decades ago and rejuvenated as successful lifesaving therapy but it was thought contra-indicated until it recently rectified by the authorities on $\mathrm{HN}$ [14-18,22,23,30,31,38,39]. Recognizing VOS1 has also helped in recognizing VOS2 that resolved the puzzle of the acute respiratory distress syndrome (ARDS) [21].

VOS2 is induced by sodium-based fluids such as crystalloids of normal saline and Hartman's solution, colloids such as plasma and plasma substitutes and blood. Although better tolerated it induce VOS2 without serological markers, affirming the concept of VOS but it remains disbelieved. A normal daily intake of 3.5 litres (L) of fluid causes signs when intravenously infused over 2-3 h but can be a serious gain in $<1 \mathrm{~h}$. Why is it so difficult to recognize these facts on encountering serious cases even of VOS 1 that is characterized with HN? Although the systemic and bizarre signs of severe TURP syndrome are well documented in case reports, it is extremely difficult to relate to $\mathrm{VO} / \mathrm{T}$ and fluid type, even on monitoring the gained volume, measuring and replacing blood loss and excluding septicaemia.

The complex signs of cardiovascular disturbance of shock and MVOD/F of the TURP syndrome are very variable in severity, up to cardiac or cardiopulmonary arrest and death, with many presentation masks and differential diagnoses. Hence, when seen in the complex surgical setting, they are wrongly attributed to known causes of shock, coma, respiratory distress, renal and heart failure or arrest as signs of MVOD/F that may occur in any combinations. Of the well documented presentation masks of VOS1, one is shock apparent to anaesthetists and surgeons during or immediately after the surgery and another is coma recognised later by physicians. More important, neither the concept nor mechanism of VOS by disturbing capillary dynamics has been recognized, despite explaining the patho-aetiology of the TURP syndrome and shock, highlighting its link with MVOD/F syndrome or APRDS [14-20,21,36-38,40]. A prospective study on 100 TURP patients among whom 10 suffered the TURP syndrome demonstrated that VO is the most significant factor in its patho-etiology (Figure 6 and Table 1) [16,17]. Also a case series of 23 patients affirmed $\mathrm{VO}$ as the correct patho-aetiology of the TURP syndrome (Figure 7) [18].

Table 1: shows the multiple regression analysis of total per-operative fluid gain, drop in measured serum osmolality (OsmM), sodium, albumin, $\mathrm{Hb}$ and increase in serum glycine occurring immediately post-operatively in relation to signs of the TURP syndrome. Volumetric gain and hypo-osmolality are the only significant factors. Volumetric gain is most highly significant. (Reproduced with permission of the author and Editor of BJU Int.; reference 16)

\begin{tabular}{|l|c|c|c|c|c|}
\hline Parameter & Value & Std. Err & Std. Value & T Value & P \\
\hline Intercept & & & 0.773 & & \\
\hline Fluid Gain (1) & 0.847 & 0.228 & 1.044 & 3.721 & 0.0001 \\
\hline Osmolality & 0.033 & 00.014 & -0.375 & 2.42 & 0.0212 \\
\hline Na+ (C_B) & 0.095 & 0.049 & 0.616 & 1.95 & 0.0597 \\
\hline Alb (C_B) & 0.062 & 0.087 & 0.239 & 0.713 & 0.4809 \\
\hline Hb (C_B) & -0.282 & 0.246 & -0.368 & 1.149 & 0.2587 \\
\hline Glycine (C_B) & $-4.973 \mathrm{E}-5$ & $5.975 \mathrm{E}-5$ & -0.242 & 0.832 & 0.4112 \\
\hline
\end{tabular}

There is no doubt that the erroneous Starling's law is responsible for the induction of the two newly discovered VOS. Volumetric overload shocks (VOS) are iatrogenic complications of fluid therapy in hospitals [17-19]. It is common but overlooked and underestimated. When it is realized that acute dilution hyponatraemia (HN) and ARDS is representative of each type of the two VOS it would be appreciated that it has staggering morbidity, cost and mortality. It affects hundreds of thousands of patients worldwide each year including surgical, urological and obstetric patients of men, women and children undergoing surgery. It concerns new discoveries in medicine and physiology [43]. This article brings these new discoveries into the attention of readers, particularly surgeons and anaesthetists as these condition concerns them most.

Other scientific discoveries in addition to the 2 VOS are proving the physiological law of Starling for the capillary-interstitial fluid transfer wrong and finding a new correct replacement which is the hydrodynamic of the porous orifice $(\mathrm{G})$ tube (Figure 3 ) as presented here. Starling's law being wrong has resulted in many errors and misconceptions on fluid therapy during prolonged surgery and the resuscitation of shock and the acutely ill patients. This misleads physicians into giving too much fluid which induces VOS, causing cardiac arrest or respiratory arrest or both "cardiopulmonary arrest" immediately in theatre or ARDS later $[20,21,37,44,45,49,50]$.

VOS are two types depending on the type of fluid: VOS 1 is induced by sodium-free fluid such as 5\% Glucose and/or 1.5\% Glycine used 
as irrigating fluid during the transurethral resection of the prostate (TURP) surgery. It is known in urology as the TURP syndrome or hyponatraemic shock $[16,31,36]$.

This VOS 1 is induced by $1.5 \%$ Glycine absorption and 5\% glucose infusion of about 3.5-5 liters or $>5 \%$ of body weight and is characterized with dilution hyponatraemia (HN) [46-48]. Hyponatraemia has 2 nadirs and 2 paradoxes making it dynamic and illusive [46,47]. The 2 nadirs are: The immediate drop of serum sodium level as result of dilution of the extra-cellular fluid that occurs during or immediately after surgery. The second nadir is that occurring later within 24 hours after water shift into the intracellular compartment causing spontaneous elevation of serum sodium level towards normal, yet the clinical picture gets worse due to generalized cellular oedema. This cellular oedema manifests as MVOD/F syndrome, ARDS or AKI. The 2 paradoxes are: A pathological volumetric overload induces hypotensive shock of VOS and acute renal failure (ARF) or AKI which is paradoxical to the response of physiological volume replacement that treats hypotensive shock and induces diuresis.

VOS1 currently has a lifesaving therapy of hypertonic sodium therapy (HST) of $5 \% \mathrm{NaCl}$ or $8.4 \% \mathrm{Co} 3$ [48]. It may present with cardiopulmonary arrest or one or more of the other manifestations of MVOD/F syndrome- being the new name for ARDS $[40,41,44]$. The clinical manifestations include in addition to cardiorespiratory features: coma with convulsions and paralysis, AKI and hepatic dysfunction. It also causes coagulopathies and excessive bleeding at the surgical site [34]. VOS1 affects women too during the transcervical resection of endometrium due to $1.5 \%$ Glycine absorption, and during Caesarean section due to excessive 5\% Glucose infusion $[37,38,39,44]$.

VOS is always mistaken for one of the recognized shocks such as haemorrhagic and septic shocks hence wrongly treated with further volume expansion using sodium-based isotonic fluids. This induces VOS2 and cardiopulmonary arrest that has no serum markers of HN and causes ARDS in patients who survive a little longer [45]. Multiple regression analysis has proved that volumetric overload is the most significant factor in causing the clinical picture of VOS (Figures 6 and 7 and Table 1) [16]. Cardiac arrest or respiratory arrest in isolation, or a combination of both have been reported as immediate presentation of the TURP syndrome induced by $1.5 \%$ Glycine and dilution hyponatraemia induced by $5 \%$ Glucose during surgery as representative of VOS1 [37,38,39,44,49,50].

Volumetric overload shock type 2 (VOS2) is induced by massive infusion of sodium-based fluids such as normal saline, Hartmann, plasma, plasma substitutes and/or blood. VOS2 may complicate VOS1 or is induced by sodium-based fluid during fluid therapy for resuscitation of shock and the critically ill and prolonged surgery and presents with ARDS later [14-21,40]. Volumetric gain of 12-14 litres of sodium-based fluids reported in the first article on ARDS which is the only article in the whole literature, other than the articles of mine referenced here, that documents the volume of retained fluid in ARDS [40]. The question of: "Does Raising the Central Venous Pressure (CVP) in Treating Shock with Fluids Induce Volumetric Overload Shocks (VOS)?" has been positively answered [51,52]. The above mentioned discoveries should make the Medical World wake up, pay attention and listen to what this article has to say [53].

\section{Summary}

Hydrodynamic studies on a porous orifice $(G)$ tube, based on capillary ultra-structure, demonstrate results which differ from Poiseuille's in a strait uniform tube and hence challenge the role attributed to arterial pressure as a filtration force in Starling's law. A perspective literature review shows that the oncotic pressure force has been previously cancelled and the law has failed to explain the capillary-ISF transfer in most parts of the body.

A concept based on a new hydrodynamic phenomenon is proposed for the capillary-ISF circulation. It explains this vital circulation in every organ and tissue under both physiological and pathological conditions. A rapid autonomous dynamic magnetic field-like $\mathrm{G}-\mathrm{C}$ circulation occurs between fluid in the $\mathrm{G}$ tube's lumen and a surrounding fluid compartment $\mathrm{C}$. Based on results of studies on a circulatory model incorporating the $\mathrm{G}-\mathrm{C}$ apparatus, factors which initiate, regulate and affect the $\mathrm{G}-\mathrm{C}$ circulation, its physiological and haemodynamic relevance and its clinical importance to the pathogenesis of oedema, shock and MVOD/F syndrome or ARDS and $\mathrm{AKI}$ are given.

The presented evidence does not only prove that Starling's law is wrong, but also provide the correct replacement; the hydrodynamic of the $G$ tube explaining the capillary-ISF circulation in every organ and tissue of the bogy. Also the physiological evidence on capillary working as G tube not Poiseuille's tube is provided. The presented evidence proves without a doubt that Starling's law is wrong and provide its correct replacement of the $\mathrm{G}$ tube hydrodynamic. This erroneous law is responsible for the many errors and misconception prevailing in fluid therapy during resuscitation of shock and acutely ill patients misleading physicians into giving too much fluid and inducing VOS causing MVOD/F or ARDS and AKI.

VOS are common iatrogenic complication of fluid therapy in hospitals that is overlooked and underestimated. It may present in theatre as cardiopulmonary arrest or later with coma and ARDS. VOS is 2 types; VOS 1 and VOS2. VOS1 is induced by 3.5-5 litres of sodium-free fluid and is characterized with dilution HN that has 2 nadirs and 2 paradoxes, is most dynamic and illusive and currently has a lifesaving therapy of HST. VOS2 may complicate VOS1 or occur de novo complicating sodium based fluid therapy during resuscitation of shock, acutely ill patients and prolonged surgery. It has no obvious serological markers or none. Many errors and misconceptions mislead physicians into giving too much fluid for resuscitation due to faulty rules on fluid therapy dictated by the wrong Starling's law. The correct replacement for this law is the hydrodynamic of the porous orifice $(G)$ tube. These scientific discoveries should make the Medical World wake up, pay attention and listen to what this article has to say.

\section{References}

1. Starling EH (1886) Factors involved in the causation of dropsy. Lancet 2: 1266-1270, 1330-1334 and 1406-1410.

2. Folkow B, Neil E (1971) Oxford University Press: London. Circulation 1-125.

3. Rhodin JA (1967) The ultra structure of mammalian arterioles and precapillary sphincters. J Ultrastructure Research 18: 181 222.

4. Karnovsky MJ (1967) The ultra structural basis of capillary permeability studied with peroxidase as a tracer. J Cell Biol 35: 213-236. 
5. Hendry EB (1962) The osmotic pressure and chemical composition of human body fluids. Clinical Chemistry 8: 246265.

6. Guyton AC, Coleman TG (1968) Regulation of interstitial fluid volume and pressure. Annals New York Academy of Sciences 150: 537-547.

7. Calnan JS, Pflug JJ, Chisholm GD, Taylor LM (1972) Lymphatic surgery. Proceedings Royal Soc Med 65: 715-719.

8. Cochrane Injuries Group (1998) Human albumin administration in the critically ill patients: systemic review of randomized controlled trials: Why albumin may not work. BMJ 317: 235240.

9. Keele CA, Neil E, Joels N (1982) Sampson Wright Applied Physiology. 13th ed. Oxford University Press. Oxford.

10. Renkin EM (1986) Some consequences of capillary permeability to macromolecules: Starling's hypothesis reconsidered. Am J Physiol (Heart Circ Physiol); 250: H706-H710.

11. Ghanem AN (2001) Magnetic field-like fluid circulation of a porous orifice tube and its relevance to the capillary-interstitial fluid circulation: preliminary report. Medical Hypotheses 56: 325-334.

12. Ghanem KA and Ghanem AN (2017) The proof and reasons that Starling's law for the capillary-interstitial fluid transfer is wrong, advancing the hydrodynamics of a porous orifice $(\mathrm{G})$ tube as the real mechanism. Blood, Heart and Circ 1: 1-7.

13. Ghanem KA, Ghanem AN (2017) The Physiological Proof that Starling's Law for the Capillary-Interstitial Fluid Transfer is wrong: Advancing the Porous Orifice $(G)$ Tube Phenomenon as Replacement. Open Acc Res Anatomy 1:1-7.

14. Ghanem AN, Ghanem SA (2016) Volumetric Overload Shocks: Why Is Starling's Law for Capillary Interstitial Fluid Transfer Wrong? The Hydrodynamics of a Porous Orifice Tube as Alternative. Surgical Science 7: 245-249.

15. Ghanem AN (1988) The Transurethral Prostatectomy (TURP) Syndrome: An Investigation of the Osmotic and Metabolic Sequelae of Volumetric Overload. MD Thesis. Institute of Urology and Nephrology, Mansoura University, Mansoura, Egypt.

16. Ghanem AN, Ward JP (1990) Osmotic and metabolic sequelae of volumetric overload in relation to the TURP syndrome. $\mathrm{Br}$ J Urol 66: 71-78.

17. Ghanem SA, Ghanem KA, Ghanem AN (2017) Volumetric Overload Shocks in the Patho-Etiology of the Transurethral Resection of the Prostate (TURP) Syndrome and Acute Dilution Hyponatraemia: The Clinical Evidence Based on Prospective Clinical Study of 100 Consecutive TURP Patients. Surg Med Open Access J 1:1-7.

18. Ghanem KA and Ghanem AN (2017) Volumetric overload shocks in the patho-etiology of the transurethral resection prostatectomy syndrome and acute dilution hyponatraemia: The clinical evidence based on 23 case series. Basic Research Journal of Medicine and Clinical Sciences 6: 35-43.

19. Ghanem AN, Ghanem SA (2016) Volumetric Overload Shocks: Why Is Starling's Law for Capillary Interstitial Fluid Transfer Wrong? The Hydrodynamics of a Porous Orifice Tube as Alternative. Surgical Science 7: 245-249.

20. Ghanem AN (2018) The Adult Respiratory Distress Syndrome: Volumetric Overload Shocks in Patho-Etiology Correcting Errors and Misconceptions on Fluid Therapy, Vascular and Capillary Physiology. Surg Med Open Acc J 2.

21. Ghanem AN (2020) Volumetric overload shocks (VOS) causing the acute respiratory distress syndrome (ARDS): The Complete Evidence. EC Emergency Medicine and Critical Care 4.2: 01-08.

22. Guyton AC (1986) Textbook of Medical Physiology. An HBJ International Seventh Edn. WB Saunders Company. Philadelphia London. 19: 221.

23. Ghanem AN (1985) Hypoalbuminaemic hyponatraemia: a new syndrome. Br Med. Jour 29: 1502.

24. Ghanem AN. Wojtulewski JA, Penney MD (1987) Dangers in treating hyponatraemia. Br Med J 294: 837.

25. Ghanem AN (1988) Hyponatraemia and hypo-osmolality. Lancet 2: 572 .

26. Ghanem AN, Ward JP (1988) Fluid absorption during urological surgery. Br J Uro 61: 168-169.

27. Ghanem AN (1999) Frequency and causes of fluid absorption: a comparison of the three techniques for resection of the prostate under continuous pressure monitoring. BJU Int 84: 891-892.

28. Ghanem AN (2000) Monitoring the critically ill patient. J R Coll Surg Edinb 45: 138-139.

29. Ghanem AN (2002) Hyponatraemia during and after TUR prostatectomy. Saudi Med J 23: 477-479.

30. Pepe PE (2003) Editorial: Shock in polytrauma. Br Med J 327: 1119-1120.

31. Harrison III RH, Boren JS, Robinson JR (1956) Dilutional hyponatraemic shock: another concept of the transurethral prostatic reaction. J Uro 75: 95-110.

32. Danowski TS, Winkler AW, Elkington JR (1946) The treatment of shock due to salt depression; comparison of isotonic, of hypertonic saline and of isotonic glucose solutions. J Clin Invest 25: 130.

33. Spencer Hoyt H, Goebel J L, Lee HI, Schoenbrod J (1958) Types of shock reaction during transurethral resection and relation to acute renal failure. J Uro 79: 500-507.

34. Bird D, Slade N, Feneley RCL (1982) Intravascular complication of transurethral prostatectomy. Br J Uro 54: 564-565.

35. Dandonna P, Fonseca V, Baron (1985) Hypoalbuminaemic hyponatraemia: a new syndrome? Br Med Jour 291: 1253-1255.

36. Hahn RG (1997) Irrigating fluids in endoscopic surgery. Br J Uro 79: 669-680.

37. Arieff AI, Ayus JC (1993) Endometrial ablation complicated by fatal hyponatraemic encephalopathy. JAMA 270: 1230-1232.

38. Arieff AI (1986) Hyponatraemia, convulsion, respiratory arrest and permanent brain damage after elective surgery in healthy women. N Engl J Med 314: 1529-1534.

39. Arieff AI (1993) Management of hyponatraemia. Br Med Jour 307: 305.

40. Ashbaugh DG, Bigelow DB, Petty TL, Levine BE (1967) Acute respiratory distress in adults. Lancet 2: 319-323.

41. Webster NR (1999) Monitoring the critically ill patient. J R Coll Surg Edin 44: 386-393.

42. Watters DAK, Chamroonkul MA, Eastwood MA (1984) Changes in liver function associated with parentral nutrition. J Roy Coll Surg Edin 29: 339-344.

43. Ghanem AN (2019) Ghanem's New Discoveries in Medicine, Physiology and Urology and Nephrology? Exp Tech Urol Nephrol 2.

44. Sarah N Bernstein, Gaston A Cudemus-Deseda, Vilma E Ortiz, Annekathryn Goodman, Arminder S Jassar (2019) CASE RECORDS OF THE MASSACHUSETTS GENERAL HOSPITAL. Case 33-2019: A 35-Year-Old Woman with Cardiopulmonary Arrest during Cesarean Section. N Engl J 
Med 381: 1664-1673.

45. Ghanem AN (2020) Cardiac arrest and volumetric overload shocks (VOS) complicating fluid therapy. EC Clinical and Medical Case Reports. (In the press)

46. Ghanem AN (2018) Short Communication. Hyponatraemia: Nadirs and Paradoxes of the Missing Volumetric Overload. Open Access Journal of Surgery 10.

47. Ghanem AN, Ghanem SA, Ghanem KA, Pindoria N, Elsayed YS (2019) Illusive Dynamic Nadirs and Masks of Postoperative Hyponatraemia and the TURP Syndrome: Volumetric overload over time $(\mathrm{VO} / \mathrm{T})$ concept for resolving its puzzle. JOJ uro \& nephron 6: 555691.

48. Ghanem AN (2018) Therapy of Hyponatremia: End of Era or Minority Report? Biomed J Sci \& Tech Res 11: 8645-8648

49. Charlton AJ (1980) Cardiac arrest during transurethral surgery after absorption of 1.5\% glycine. Anaesth 35: 804-807.

50. Jacobson J (1965) Prolonged respiratory inadequacy following Transurethral Resection of the Prostate. Anaesth 20: 329-333.

51. Ghanem AN (2019) Does Raising the Central Venous Pressure (CVP) in Treating Shock with Fluids Induce Volumetric Overload Shocks (VOS)? Adv Card Res 1: 2019.

52. Ghanem AN (2018) Volumetric Overload Shocks: Resolving the puzzles of the transurethral resection of the prostate (TURP) syndrome, acute dilution hyponatraemia $(\mathrm{HN})$ and the acute respiratory distress syndrome (ARDS). Scholars Press USA.

53. Ghanem AN (2019) Medical World wake up, pay attention and listen: Ghanem's new scientific discoveries in Medicine, Physiology, Urology, Nephrology, Cardiovascular and Surgery. EC Clinical \& Medical Case Reports 2.9: 01-06.
Copyright: (02020 Ahmed N. Ghanem. This is an open-access article distributed under the terms of the Creative Commons Attribution License, which permits unrestricted use, distribution, and reproduction in any medium, provided the original author and source are credited. 